Marquette University

e-Publications@Marquette

Mechanical Engineering Faculty Research and

Publications

Mechanical Engineering, Department of

$1-1-2018$

\title{
A Degradation Function Consistent with Cocks-Ashby Porosity Kinetics
}

John A. Moore

Marquette University, john.moore@marquette.edu

Accepted version. International Journal of Fracture, Vol 209, No. 1/2 (January 2018): 231-234. DOI. (C) 2017 Springer Science+Business Media B.V. Used with permission.

John A. Moore was affiliated with Lawrence Livermore National Laboratory at the time of publication. 
Marquette University

e-Publications@Marquette

\title{
Mechanical Engineering Faculty Research and Publications/College of Engineering
}

This paper is NOT THE PUBLISHED VERSION; but the author's final, peer-reviewed manuscript. The published version may be accessed by following the link in th citation below.

International Journal of Fracture, Vol. 209, No. $1 / 2$ (January 2018): 231-234. DOI. This article is (C) Springer and permission has been granted for this version to appear in e-Publications@Marquette. Springer does not grant permission for this article to be further copied/distributed or hosted elsewhere without the express permission from Springer.

\section{A Degradation Function Consistent with Cocks-Ashby Porosity Kinetics}

\author{
John A. Moore
}

Lawrence Livermore National Laboratory, Livermore, CA

The load carrying capacity of ductile materials degrades as a function of porosity, stress state and strain-rate. The effect of these variables on porosity kinetics is captured by the Cocks-Ashby model; however, the CocksAshby model does not account for material degradation directly. This work uses a yield criteria to form a degradation function that is consistent with Cocks-Ashby porosity kinetics and is a function of porosity, stress state and strain-rate dependence. Approximations of this degradation function for pure hydrostatic stress states are also explored.

Degradation function; Porosity; Damage; Ductile fracture; Strain-rate dependence

\section{Introduction}

The load carrying capacity of ductile materials degrades as a function of porosity (Vaz and Andrade Pires [14] ). However, this degradation is also a function of stress state (Benzerga et al. [3] ) and strain-rate (Rodriguez et al. [12] ). Gurson's model (Gurson [7] ) for ductile materials accounts for this degradation through a yield criteria that is an explicit function of stress state and porosity. Gurson's model, however, accounts for strain-rate 
dependence only implicitly, as Gurson's yield criteria is function of flow stress, and flow stress may be strain-rate dependent.

For strain-rate dependent problems, the Cocks-Ashby model (Cocks and Ashby [5] ) provides a porosity evolution (kinetics) expression that is a function of porosity, stress state, and strain-rate exponent. However, their model does not give a corresponding yield criteria to evaluate material degradation. Inversely, since porosity evolution is related to a yield behavior, Cocks-Ashby's porosity evolution expression does not necessary correspond with any general yield criteria. This work proposes a degradation function that is consistent with Cocks-Ashby's porosity kinetics and gives thoughts on its implementation and uses.

\section{Background}

Gurson's model gives a yield criteria $\phi\left(\sigma_{h} / \bar{\sigma}, f, m\right)=0$, where $\sigma_{h}$ is hydrostatic stress, $\bar{\sigma}$ is flow stress, $f$ is porosity, and $m$ is strain-rate exponent. Strain-rate exponent characterizes the power-law relation between stress and strain-rate $(\varepsilon)=\varepsilon_{o}\left(\sigma_{e q} / \sigma_{o}\right)^{1} / m$ where $\dot{\varepsilon}$ is the rate of small strain evolution, $\sigma_{e q}$ is equivalent stress, and $\varepsilon_{o}, \sigma_{o}$ are material parameters.

Combining mass conservation with the flow rule for plastic strain gives a relation between porosity evolution and Gurson's (or any) yield criteria,

$$
f=(1-f) \lambda \operatorname{tr}(\partial \phi / \partial \sigma \mathrm{ij}),(1)
$$

where $\dot{\lambda}$ is a strain rate multiplier and $\sigma$ is the Cauchy stress. Cocks-Ashby's porosity evolution expression is given by:

$\dot{f}=c_{1} \sinh \left\{c_{2} \frac{2-m}{2+m}\left(\frac{\sigma_{h}}{\sigma_{e q}}\right)\right\}\left(\frac{1}{(1-f)^{1 / m}}-(1-f)\right) \dot{\bar{\varepsilon}} p,(2)$

where $\dot{\bar{\varepsilon}} p$ is the effective plastic strain rate and Cocks-Ashby's original form has been modified to include two calibration parameters $c_{1}$ and $c_{2}$. Equation (2) does not adhere to Eq. ( 1 ) and (possibly as a result) is used in crystal scale calculations where a yield criteria is not generally known in a functional form. In these cases, phenomenological degradation is used. Specifically, Kweon ([8] ) used the degradation function $w=1-c_{3} f$ and Barton et al. ([2] ) used the degradation function $w=1-\tanh \left(c_{3} f\right)$ where $c_{3}$ was a degradation parameter independent of Eq. (2). In both cases $\dot{\bar{\varepsilon}} p$ in Eq. (2) was replaced with the sum of shearing rates in a crystal.

Several yield criteria for strain-rate dependent materials have been proposed. Marin and McDowell ([10]) expressed these criteria in the form

$\phi=\sqrt{3 h 1 J 2+\Omega}-h_{3} \bar{\sigma}=0,(3)$

where $J_{2}$ is the second invariant of deviatoric stress (related to equivalent stress by $\sigma_{e q}=\sqrt{3 J_{2}}$ ), $\Omega$ is a function to be discussed later, and $h_{1}$ and $h_{3}$ are functions of $f$ and $m$ corresponding to the various yield criteria of Cocks ([4] ), Michel and Suquet ([11] ) and Duva and Hutchinson ([6] ), or Sofronis and McMeeking ([13] ).

\section{Theory}

A general yield criteria is given as (Vaz and Andrade Pires [14] ):

$\phi=\sigma_{e q}-w \bar{\sigma}=0 .(4)$ 
Marin and McDowell ([10] ) express porosity evolution in terms of the yield criteria in Eq. (3) as

$\dot{f}=\frac{1-f}{\sqrt{h_{1} 2 J_{2}}} \frac{\partial \Omega}{\partial I 1} \dot{\bar{\varepsilon}} p,(5)$

where $I_{1}$ is the first invariant of stress.

Marin and McDowell ([10] ) equate this to Cocks-Ashby's porosity evolution expression by assuming that $\sinh (x) \approx x$ and integrating with respect to 11 to yield ${ }^{1}[\underline{1}]$ :

$\Omega=h_{1}\left(\frac{2}{9}\right)\left(\frac{2-m}{2+m}\right) I_{1}^{2}\left(\frac{1}{(1-f)^{\frac{1}{m}+1}}-1\right)$ (6)

The yield criteria in Eq. (3) can be written in terms of a degradation function (as in Eq. 4) if $\Omega$ can be written as $\Omega=3 J_{2} \Omega^{*}$. Thus, Eq. (6) could be multiplied by $\left(3 J_{2} / 3 J_{2}\right)$ to achieve this form. However, a more natural and general solution is to integrate Eq. (5) exactly without the small sinh assumption to give:

$\Omega=\left(3 J_{2}\right) h_{1} \frac{(2+m)}{(2-m)} \cdot \cosh \left[\frac{2}{3} \frac{(2-m)}{(2+m)} \frac{I_{1}}{\sqrt{3 J_{2}}}\right]\left(\frac{1}{(1-f)^{\frac{1}{m}+1}}-1\right),(7)$

using $\Omega=3 J_{2} \Omega^{*}, \sigma_{e q}=\sqrt{3 J_{2}}, \sigma_{h}=I_{1} / 3$ and including the two calibration parameters $c_{1}, c_{2}$ gives:

$\Omega^{*}=\frac{c_{1}}{c_{2}} 2 h_{1} \frac{(2+m)}{(2-m)} \cdot \cosh \left[c_{2} \frac{(2-m)}{(2+m)} \frac{\sigma_{h}}{\sigma_{e q}}\right]\left(\frac{1}{(1-f)^{\frac{1}{m}+1}}-1\right)$. (8)

With this modification, Eq. (3) can be written as:

$\sigma_{e q}-\frac{h_{3}}{\sqrt{h_{1}+\Omega^{*}}} \bar{\sigma}=0,(9)$

which fits in the form $\phi=\sigma_{e q}-w \bar{\sigma}$ and gives a degradation function consistent with Cocks-Ashby porosity kinetics:

$w=\frac{h_{3}(f, m)}{\sqrt{h_{1}(f)+\Omega^{*}\left(f, m, \sigma_{h}\right)}} .(10)$

Equation (10) is plotted for several strain-rate exponents in Fig. 1 highlighting the explicit dependence of degradation on m absent from Gurson's model. 


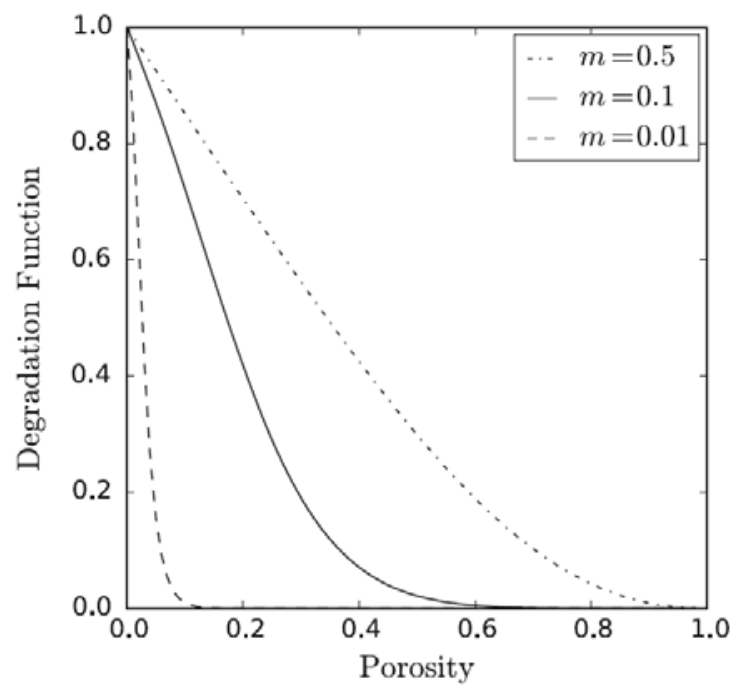

Figure 1. The degradation function's porosity dependence for several strain-rate exponents and a triaxiality of 0.3

This is simply a reinterpretation of the general yield criteria in Marin and McDowell ([10]) (neglecting only the small sinh assumption), but it gives physical insight into the degradation of the material, and gives a functional form for degradation that both captures strain-rate dependence explicitly and is consistent with Cocks-Ashby porosity kinetics.

\section{Implementation}

This section implements Eqs. (2) and (4) using the new degradation function in Eqs. (8) and (10), and gives some thoughts on a useful approximation for Eq. (8). These expressions are implemented in the ale3d finite element code developed at Lawrence Livermore National Laboratory (Anderson et al. [ 1] ). Simulations are executed on a single 3D finite element loaded with a constant uniaxial velocity and no lateral constraints. Cocks ([4] ) yield criteria is used giving $h_{1}=1+(2 / 3) f$ and $h_{3}=(1-f)^{1 /(1+m)}$. Kirchhoff's stress $\tau_{i j}$ is used in evaluation of the yield criteria, and is converted to Cauchy stress via $\tau_{i j}=J_{\sigma_{i j}}$, where $J=1 /(1-f)$, for calculation of internal forces. A Swift-type flow stress expression $\bar{\sigma}=\bar{\sigma} o\left(1+\bar{\varepsilon}^{p} / e_{o}\right)^{n S}$ is used with parameters set to $\bar{\sigma}_{o}=$ $260 \mathrm{MPa}, e_{o}=0.004, n_{S}=0.1$. The Young's modulus and Poisson's ratio are $73.3 \mathrm{GPa}$ and 0.33 respectively; The calibration parameters are $c_{1}=0.1, c_{2}=0.5$; The strain-rate exponent is $m=0.1$, where strain-rate is considered constant; and Initial porosity is 0.07 for the simulation without nucleation.

It is of consequence for implementation that Eq. (2) is singular for pure hydrostatic stress (i.e., $\sigma_{e q}=0$ ). The appendix of Cocks and Ashby ([5] ) accounts for this by providing a separate $\dot{f}$ expression for pure hydrostatic tension conditions; however, this expression requires both an ad-hoc barrier between high triaxialities (that approach pure hydrostatic stress) and low triaxialities as well as implementation of a mechanism for switching between cases. 


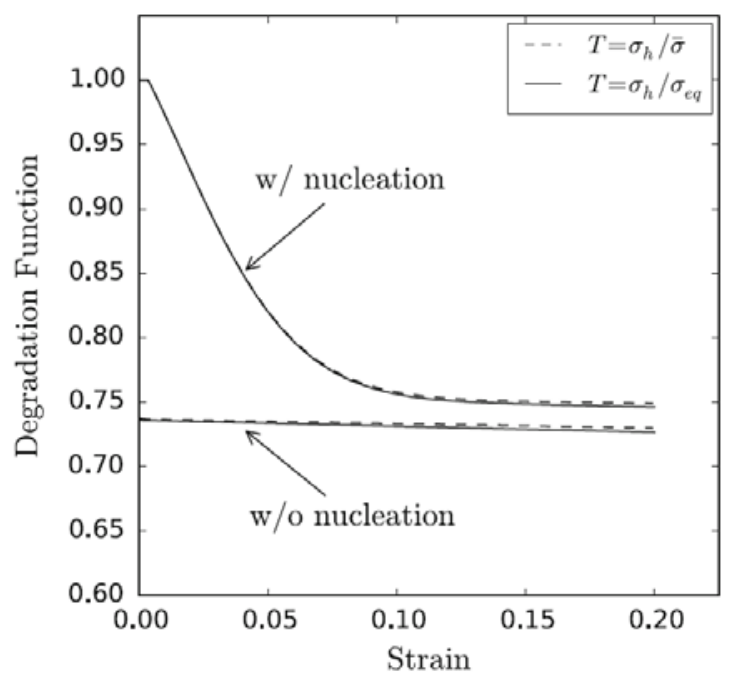

Figure 2. The degradation function with different approximation of triaxiality $(T)$. When nucleation is present no initial porosity is prescribed

To protect against singularity for pure hydrostatic stress states, this work approximates the triaxiality $(T=$ $\left.\sigma_{h} / \sigma_{e q}\right)$ in Eqs. (2) and (8) by replacing oeq with $\bar{\sigma}$. The same approximation was made by Lee and Dawson ([9]) and was motivated by their observation that $\sigma e q$ is "very close" to $\bar{\sigma}$ for rate-independent inelastic materials. The effect of this approximation on degradation (under a uniaxial load) with and without porosity nucleation are shown in Fig. 2. The scenario with nucleation has no initial porosity and porosity nucleation is defined by:

$\dot{f}_{N}=\frac{f_{N}^{o}}{s_{N} \sqrt{2 \pi}} \exp \left[-\frac{1}{2}\left(\frac{\bar{\varepsilon}-\varepsilon_{N}}{s_{N}}\right)^{2}\right] \dot{\bar{\varepsilon}} p \cdot(11)$

In Eq. (11), the porosity available for nucleation is $f_{N}^{o}=0.003$, the reference strain is $\varepsilon_{N}=0.01$, and the standard deviation of strain-based nucleation equivalent plastic strain is $s_{N}=0.001$.

Figure 2 shows that, while Eq. (10) allows for increasing degradation (with finite initial porosity) before the onset of plastic strain, this elastic degradation has minimal effect (and is barely distinguishable in Fig. 2). Figure 2 also shows that approximating triaxiality using $\bar{\sigma}$ results in negligible error for the loading and parameters studied.

\section{Conclusions}

This work shows that the general yield criteria outlined by Marin and McDowell ([10] ) can be expressed as a degradation function. This degradation function has the following advantages over its predecessors: It.

- gives physical insight into the degradation of the material which may be opaque from a yield criteria;

- is both consistent with Cocks-Ashby porosity kinetics and an explicit function of strain-rate exponent and stress state;

- is useful for crystal scale (i.e., crystal plasticity) problems where a yield function is not used directly.

Substituting $\bar{\sigma}$ for $\sigma_{e q}$ in the degradation function protected against a singularity at pure hydrostatic stress and resulted in negligible error for the loading and parameters studied 


\section{Acknowledgements}

The author would like to thank Nathan Barton for his input and discussions regarding this work. This work was performed under the auspices of the U.S. Department of Energy by Lawrence Livermore National Laboratory under Contract DE-AC52-07NA27344 (LLNL-JRNL-730381).

\section{References}

\section{Citations}

1 Anderson A, Cooper R, Neely R, Nichols A, Sharp R, Wallin B (2003) Users manual for ALE3D-an arbitrary Lagrange/Eulerian 3D code system. Technical report UCRL-MA-152204, Lawrence Livermore National Laboratory., https://wci.llnl.gov/simulation/computer-codes/ale3d

2 Barton N, Rhee M, Li S, Bernier J, Kumar M, Lind J, Bingert J (2014) Using high energy diffraction microscopy to assess a model for microstructural sensitivity in spall response. In: Journal of Physics: conference series, vol 500, IOP Publishing, p 112007

3 Benzerga A, Besson J, Pineau A, Coalescence-controlled anisotropic ductile fracture, Trans Am Soc Mech Eng J Eng Mater Technol, 1999, 121, 221, 229, 10.1115/1.2812369

4 Cocks A, Inelastic deformation of porous materials, J Mech Phys Solids, 1989, 37, 6, 693, 715, 10.1016/00225096(89)90014-8

5 Cocks A, Ashby M, Intergranular fracture during power-law creep under multiaxial stresses, Metal Sci, 1980, $14,8-9,395,402,10.1179 / 030634580790441187$

6 Duva J, Hutchinson J, Constitutive potentials for dilutely voided nonlinear materials, Mech Mater, 1984, 3, 1, 41, 54, 10.1016/0167-6636(84)90013-9

7 Gurson AL, Continuum theory of ductile rupture by void nucleation and growth: Part I-yield criteria and flow rules for porous ductile media, J Eng Mater Technol, 1977, 99, 1, 2, 15, 10.1115/1.3443401

8 Kweon S, Damage at negative triaxiality, Eur J Mech A Solids, 2012, 31, 1, 203, 212, 10.1016/j.euromechsol.2011.02.005

9 Lee YS, Dawson PR, Modeling ductile void growth in viscoplastic materials-Part I: void growth model, Mech Mater, 1993, 15, 1, 21, 34, 10.1016/0167-6636(93)90076-4

10 Marin E, McDowell D, Associative versus non-associative porous viscoplasticity based on internal state variable concepts, Int J Plast, 1996, 12, 5, 629, 669, 10.1016/S0749-6419(96)00023-X

11 Michel J, Suquet P, The constitutive law of nonlinear viscous and porous materials, J Mech Phys Solids, 1992, 40, 4, 783, 812, 10.1016/0022-5096(92)90004-L

12 Rodriguez OL, Allison PG, Whittington WR, Francis DK, Rivera OG, Chou K, Gong X, Butler T, Burroughs JF, Dynamic tensile behavior of electron beam additive manufactured Ti6Al4V, Mater Sci Eng A, 2015, 641, 323, 327, 10.1016/j.msea.2015.06.069

13 Sofronis P, McMeeking R, Creep of power-law material containing spherical voids, J Appl Mech, 1992, 59, 2S, $\mathrm{S} 88, \mathrm{~S} 95,10.1115 / 1.2899512$

$14 \mathrm{Vaz}$ M, Jr, Andrade Pires F, A note on the thermal effects upon a Gurson-type material model, Contin Mech Thermodyn, 2016, 28, 3, 785, 798, 10.1007/s00161-015-0416-7

\section{Footnotes}

1 a typographic error in Marin and McDowell (1996) gives the leading term as (2 / 8). 